\title{
Safe Piranhas: A Review of Methods and Protocols
}

Hugo Gerald Schmidt,*

Cambridge Centre for Advanced Research and Education in

Singapore, 07 RESEARCH Tower,

51 Create Way, Singapore

138602

hugo.schmidt@cares.cam.ac.uk

\section{Using Piranha Safely: An Example}

The Cambridge Centre for Advanced Research and Education in Singapore makes use of Piranha

solution in its microelectronics work. The significant risks of Piranha are addressed by this protocol, which covers the entire lifecycle of Piranha use, from acquisition to neutralization and disposal. Elements are addressed that are usually not listed in similar programs, such as the legal hazards of Piranha use and the methods and standards of training to be used. The protocol represents a practical application of the paired review of Piranha safety.

\section{INTRODUCTION}

The Cambridge Centre for Advanced Research and Education in Singapore (Cambridge CARES) is a multi-national research entity focused on carbon reduction. At CARES, Piranha solution is used to prepare glass slides for microfabrication.

Made by mixing hydrogen peroxide with sulfuric acid, Piranha solution is one of the most potent acids in existence. It reacts aggressively with all organic compounds, can start fires, and form triacetone triperoxide (TATP), the high explosive known as the 'Mother of Satan'. Even its preparation is hazardous, involving an exothermic reaction whose heat can melt or break containers or seriously injure the unwary researcher.

Further, it is classified as an explosive precursor under Singapore law [1]. The improper storage or handling of explosive precursors may be punished by a fine of SGD 100,000 and two years' imprisonment. [2]

Given the risks, care must be taken. This document lays out the procedure to equip all CARES staff to safely make, use, and neutralize Piranha solution. It is based on the extensive review of Piranha safety procedures published separately; unless otherwise specified, the source of all information in this 
procedure is derived from the review [3]. This protocol is an example the application of the review, of how general information is adapted and used in a specific circumstance.

\section{PLANNING AND PREPARATION}

Before considering any use of Piranha, the researcher should investigate whether less risky substitutes can be used, such as NoChromix, Nanostrip, Potassium hydroxide mixed with ethanol, or an activated oxygen plasma. Only when no substitute is feasible should Piranha work be considered.

Piranha is made with hydrogen peroxide, an explosive precursor regulated under the Singapore Arms and Explosive Act. Its use must be approved of in writing by the Singapore Police Force (SPF) to ensure that the act is not contravened. As of this writing, we have such approval [4].

Each user of Piranha will need to completely risk assess the work, providing a hazardous materials risk assessment, a laboratory activity risk assessment, an Explosive Precursor Risk Assessment, and a Standard Operating Procedure (SOP).

The SOP should specify the total volume of Piranha to be produced. This should be As Low As Reasonably Achievable (ALARA). This amount will be sufficient for one use and one use only. Further, no more Piranha should be produced than could be quickly neutralized with sodium bicarbonate in the event of a spill.

Under no circumstances will any batch of Piranha be stored beyond its initial use, or for more than 48 hours. One of the published cases of Piranha explosion occurred when a batch of Piranha was left unattended for a week.

In addition, the SOP should specify total volumes of hydrogen peroxide and sulphuric acid used, the ratio of the two being between $1: 3$ and 1:5. Any change in the volumes used will require a new SOP with a new approval. Approval should be obtained from the Lab Manager as well as the relevant Principle Investigators (PIs). 
All Piranha work relies on a buddy-system: Piranha workers always work in pairs. All work is conducted within normal hours. Piranha work should never be performed alone.

\section{ENGINEEING CONTROLS}

All Piranha work at Cambridge CARES is to be conducted in the CARES cleanroom. No new location will be established without consultation with the lab manager, and express, written permission from the relevant PIs and the Programme Director.

To protect workers from corrosive fumes, and to prevent the accumulation of flammable and explosive fumes, all Piranha work, from production to neutralization, will be conducted in a designated fumehood.

Only Piranha work will be carried out in the designated fume hood. All other work, including the preparation of substrates before Piranha treatment, and their drying thereafter, will be conducted away from the fume hood.

Toxic gas could be released by a Piranha spill onto toxic chemicals. No chemicals will therefore be stored in the chemical cabinet below the fume hood designated for Piranha work.

All work areas, namely, the cleanroom and the fume hood, will be properly signposted, warning all workers that Piranha is in use.

The hydrogen peroxide used in making Piranha will be stored outside the cleanroom. In line with Singapore regulations on the storage of explosive precursors, the hydrogen peroxide will be stored in a locked refrigerator under 24-hour CCTV coverage. The CCTV footage will be stored for 30 days.

As an explosive precursor, hydrogen peroxide is subject to CARES procedures for handling explosive precursors. Staff must familiarize themselves with these during the planning and preparation period. When necessary, the Piranha workers shall retrieve the hydrogen peroxide, aliquot the necessary volume in the cleanroom, and return the main bottle to the storage before continuing. 
A folder containing a copy of this safety procedure as well as the Safety Data Sheets (SDS) for hydrogen peroxide, sulphuric acid, Caro's acid, and Piranha solution will left outside the cleanroom, to be handed to emergency responders should this become necessary.

\section{EQUIPMENT FOR PIRANHA USE}

The fume hood shall contain only the equipment necessary for the preparation, use, neutralization and disposal of Piranha. These are listed in table 1.

\section{Table 1: Equipment for Piranha use}

Beaker for sulphuric acid

Beaker for hydrogen peroxide

Teflon tweezers

Pyrex Mixing dish

Pyrex crystallization dish for secondary containment
$1 \mathrm{~L}$ Wash beaker with $800 \mathrm{ml}$ DI water

$2 \mathrm{~L}$ beaker for neutralization

Pyrex waste funnel

Waste bottle with vented cap.

First- and second-level headings signal the progression of ideas in the text and help guide readers through Equipment should be arranged within the fume hood so that the workflow moves in the direction of handedness of the researcher (see figure 1). This prevents the researcher inadvertently raising an arm directly over the Piranha solution.

Clean Pyrex bottles will be used for Piranha waste; these are bottles specifically bought for the purpose. No solvent bottles will be reused. Each bottle will be washed thoroughly before use. Washing is done by filling the bottle with DI water, vigorously shaking, and emptying it. This will be repeated for a total of three times. To verify, once the washing is complete, the bottle will be filled with DI water and a sample taken to test with HPLC to ensure that the bottle does not contain any organic contamination. 

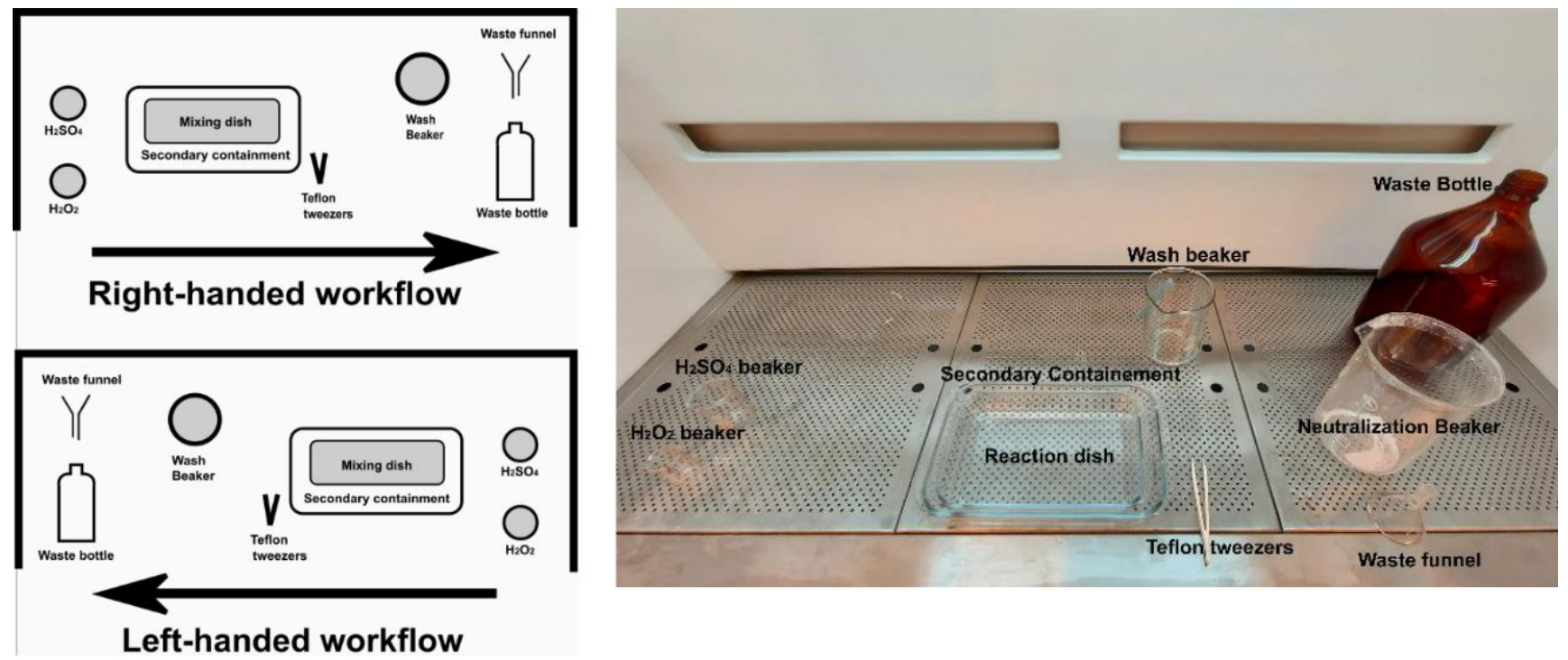

Figure S1: (left) Fume hood equipment arrangements for different workflows for different handedness, (right) Example of right-handed workflow layout in Cambridge CARES lab

An excess of sodium bicarbonate will be located nearby, more than enough to neutralize any spill.

\section{PERSONAL PROTECTICE EQUIPMENT}

Piranha solution is highly corrosive and destructive to flesh. We recommend that staff view the following video of its effects: https://www.youtube.com/watch?v=R35LI24trFc

PPE worn for Piranha use consists of a face-shield worn over safety glasses, an acid resistant apron worn over the full-body cleanroom suit, nitrile gloves below neoprene safety gloves, long trousers, and cleanroom boots. Properly suited up workers are shown in figure 2.

All staff should note the reduced dexterity from wearing neoprene gloves, and it is worth taking some time to familiarize oneself before beginning experimental work. 


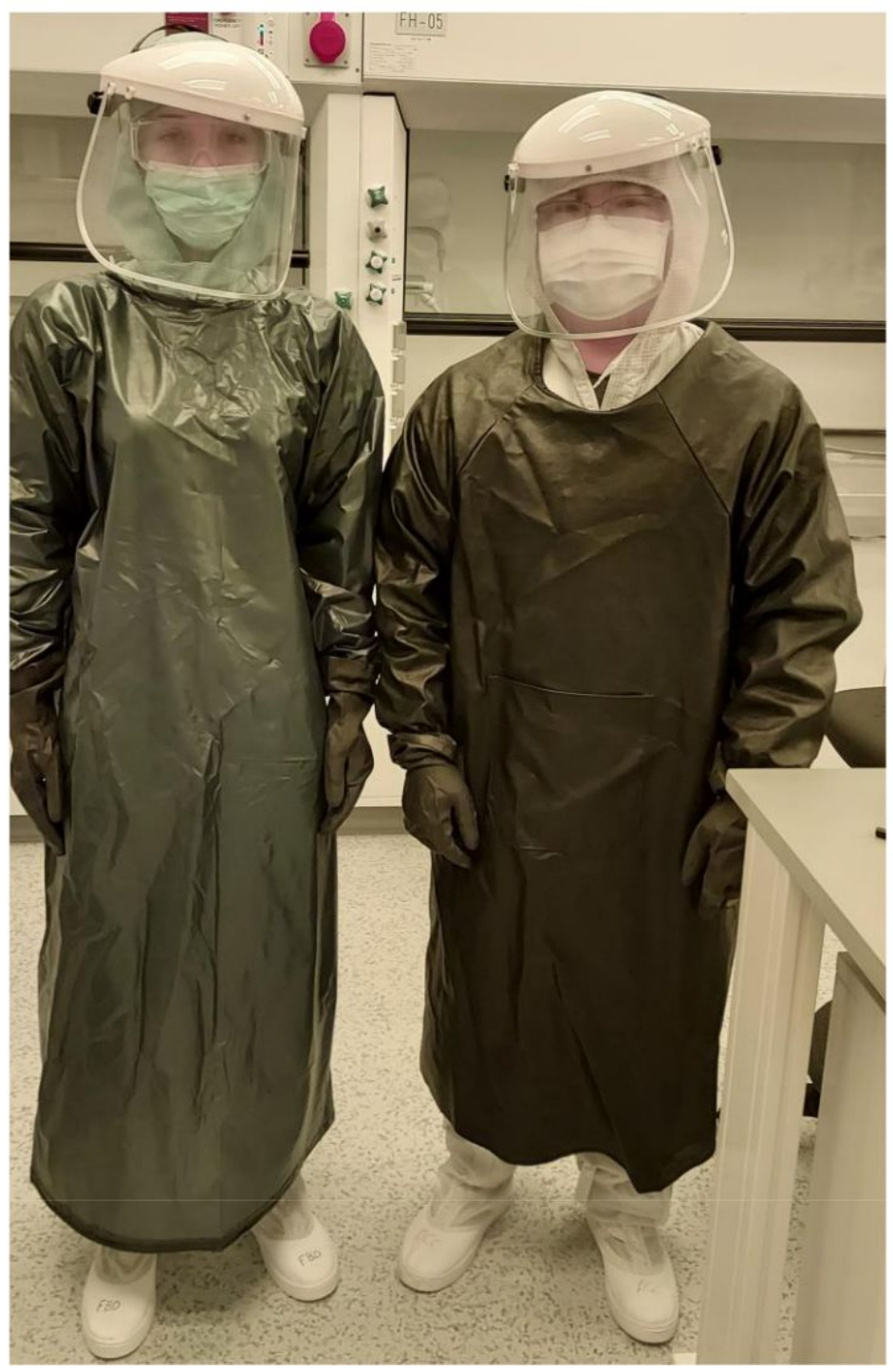

Figure S2: PPE to be worn by all Piranha users

All PPE is strictly 'second chance' protection. Any exposure to Piranha triggers immediate removal of all PPE and shutdown of activity.

PPE should be inspected before and after each use. In particular, acid aprons will be inspected for any spills, and neoprene gloves will be inspected for pinholes.

\section{PERSONAL PROTECTICE EQUIPMENT}

At CARES, a pillar of safety is awareness of 'danger signs'. This is anything - anything seen, smelt, heard or otherwise - that suggests a procedure has become unstable and that it is unsafe to proceed. For the use of Piranha solution, we have identified the following danger signs (fig. 3). 


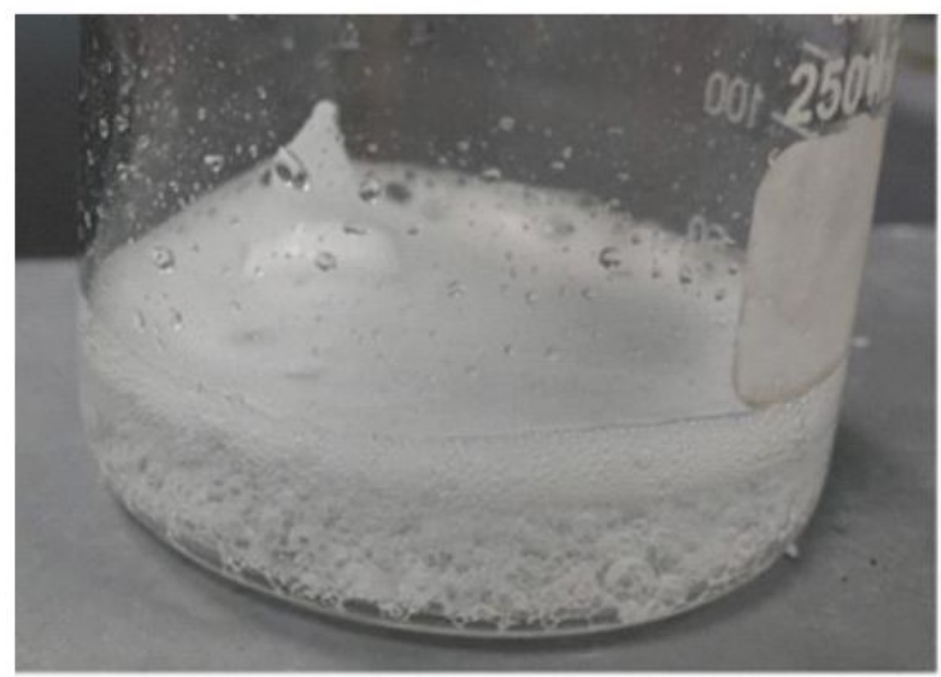

Foaming

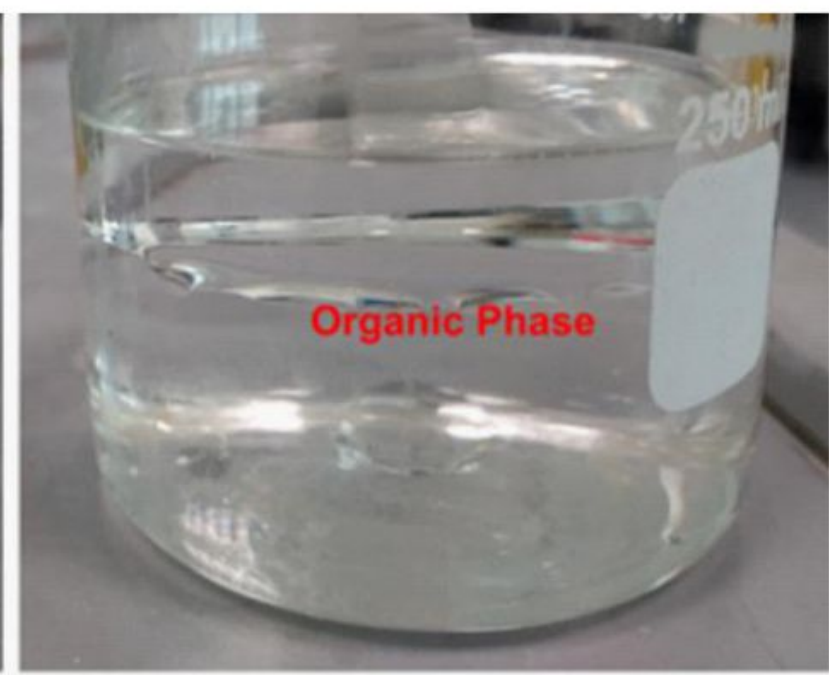

Organic Phase

Figure S3: Danger signs of Piranha requiring immediate halting of all experimental work

Smoke, spitting, foaming or other signs of an exothermic reaction

This is most likely to occur during preparation, but could occur during any stage. Response is

shutdown of all work and immediate evacuation of the cleanroom for 1 hour. Work may be resumed if the solution has calmed and there is no sign of any other trouble, in particular no formation of an organic phase.

Formation of organic phase

Formation of an organic phase (fig. S3. right) can indicate formation of TATP, with imminent danger of detonation. In this case, the cleanroom will be evacuated and locked, as will the entire CARES laboratory. Both will remain locked until the Singapore Police Force bomb squad can be contacted for safe disposal.

In particular, the waste bottle should be checked for the formation of an organic phase before each procedure is started.

PREPARING AND USING PIRANHA SOLUTION

Before beginning work, it is your responsibility to check all Piranha equipment for possible contamination with organic materials. If glassware is cleaned, it should be done with DI water only. No acetone should ever be used to clean designated Piranha tools. 
0. Piranha treatment is the last step in cleaning and preparing glass slides. Clean all slides thoroughly and dry with a nitrogen gun before any other steps, and place slides in mixing dish.

1. Retrieve hydrogen peroxide from locked refrigerator, entering the amount to be used into the log book.

2. Decant listed volume into designated beaker in the cleanroom

3. Return hydrogen peroxide to locked refrigerator.

4. Decant sulphuric acid into beaker in cleanroom.,

5. Add sulphuric acid to mixing dish, ensuring that all slides are submerged.

6. Very slowly add hydrogen peroxide to the dish, ensuring proper mixing of peroxide and acid.

7. Leave glass slides for a minimum of 1 hour while the reaction proceeds

Before continuing Piranha work, check that no organic phase has formed.

8. After ensuring that the solution has cooled sufficiently, use Teflon tweezers to retrieve glass slides.

9. Place each glass slide in wash beaker for five minutes.

10. Retrieve glass slides, dispose of wash water, refill beaker with $800 \mathrm{ml}$ DI water and replace slides for a further 5 minutes.

11. Dry glass slides outside of the designated Piranha fume hood.

Neutralizing and disposing of the solution

0. All batches of Piranha are to be disposed of immediately after use, and in general should not be stored for more than 48 hours.

1. Leave Piranha to cool overnight in the fumehood, during which time the cleanroom will be locked.

\section{Before neutralization, check that no organic phase has formed}

2. Transfer water from wash beaker to neutralization beaker. Top up until volume is 10x Piranha.

3. Carefully transfer Piranha solution to neutralization beaker.

4. Very slowly add sodium bicarbonate, stirring all the time with a glass stirring rod, to neutralize the solution.

Be aware of foaming, spitting or smoke. If these occur, stop operation and wait until the reaction

5. When sodium bicarbonate no longer dissolves but drops out of solution, check $\mathrm{pH}$ with Phenophthalein or $\mathrm{pH}$ strips. Neutral $\mathrm{pH}$ indicates neutralization is complete.

6. Examine waste bottle. Do not pick up bottle before making sure that no organic phase has formed. Sudden detonation can occur from simply agitating a TATP solution.

7. If no organic phase has formed in the waste bottle, transfer neutralized Piranha solution to waste bottle with designated funnel. Begin waste transfer by adding a little from the neutralization beaker to the bottle, and swirling it to check for bubbles or any other sign of reaction.

8. Once all neutralized Piranha has been transferred to the waste bottle, make sure that the last drops fall off the end of the funnel.

\section{During neutralization, check that no organic phase has formed in the waste bottle}

Bottles should not be filled to more than $5 \mathrm{~cm}$ below top. No bottle should be in use for more than 3 months before being disposed of with our licensed waste remover. 


\section{SPILL RESPONSE}

\section{Spills in Eyes}

Flush thoroughly with water for 15 minutes and then place ice packs on eyes. Seek immediate

emergency treatment, being sure to provide the medical staff with the Piranha folder.

\section{Spills on person}

Flush thoroughly with water for 15 minutes and seek immediate emergency treatment, being sure to provide the medical staff with the Piranha folder.

\section{Inhalation of Piranha fumes}

Remove casualty immediately to fresh air, and seek emergency treatment, being sure to provide the medical staff with the Piranha folder.

\section{Spills on floor or fume hood}

Immediately communicate the spill to the Lab Manager, and proceed with neutralization. Piranha spills must be immediately neutralized to prevent any fires or explosions from reactions.

Contain the spill in a ring of sodium bicarbonate. Then proceed to slowly sprinkle sodium bicarbonate from the outside inwards (see the training video). Foaming and reaction will occur, so be careful of spitting. Once no more reaction occurs, the spill is neutralized. Proceed as normal for a chemical spill.

Use only standard PIG absorbent pads to absorb even neutralized Piranha solution. All absorbent pads should be treated as potentially dangerous, and sealed in doubled-chemical hazard plastic bags with sufficient water to submerge the pads in case of any further reaction. The bags will be stored in a fire-proof location.

\section{TRAINING OF STAFF}

Napoleon famously required his troops to have "3 o'clock in the morning courage", the ability to take the correct course of action at any time, even under stress. At CARES, our staff are required to have "3 o'clock in the morning safety awareness". It is not sufficient that lab users merely read the safety procedures of this document, they must be able to act on them at a moments' notice - as a moment may make the difference between safety and violent detonation.

To achieve this, we operate on the principle of 'Impossible to fail, impossible to pass'. It is impossible for staff to fail these safety procedures - if researchers are unable to complete the testing perfectly, they will be given time to study the material until they do. Hence, 'impossible to fail'. 
Conversely, testing is never completed. Throughout their period of Piranha use, Piranha workers are subject to continued testing at the discretion of the Lab Manager. This is to ensure that training does not degrade over time. Hence "Impossible to pass".

Testing of competence consists of timed disrobing drills. Each Piranha user is required to be able to help their buddy disrobe, removing acid apron, gloves and face-shield, in under 10 seconds. Training further consists of an oral quiz on the significant points of this safety protocol. A perfect score is required to proceed.

\section{TRAINING QUIZ ADMINISTERED TO PIRANHA USERS}

The Lab Manager or designated representative administers this quiz. The administrator should note any ambiguities in the answers received and work to resolve them. These questions are not in order and should not be administered in the same order twice, to ensure deep understanding and immediate recall of staff.

1. What dangers are associated with Piranha use?

In particular, staff should be able to describe the multiple routes that can lead from Piranha use to 235 explosion.

2. What does PPE for Piranha consist of?

3. Describe how you put the PPE on and take it off.

In particular, staff should describe how they examine PPE for damage before and after use.

4. What ratio of hydrogen peroxide to sulphuric acid will you use?

5.Describe the different responses to spills, either spills on a person or on in the environment.

6. Where is the nearest eyewash and safety shower?

7. Describe the equipment that may be located in the Piranha fume hood.

8. How will you prepare Piranha?

Staff should stress that hydrogen peroxide is added to sulphuric acid, rather than the reverse. 
9.How will you use Piranha?

Staff should be clear that all glass slides are cleaned and dried prior to Piranha use, and that they are dried away from the Piranha fume hood after use. They should also describe how long they will wait to let the Piranha solution cool after production.

10. How do you place things in and remove things from Piranha solution?

11.How will you neutralize Piranha?

12. How do you transfer neutralized Piranha to waste bottle?

Staff should mention adding a small amount first and swirling to check for bubbles, making sure that no organic phase forms, and using a funnel and ensuring the last drops drip off at the end of transfer.

13. What are the danger signs associated with Piranha use, and what are your responses to each? Staff should describe both danger signs described in this protocol, when they are most likely to occur, what they indicate, and the appropriate responses to both.

14. Sketch the layout of our equipment in the Fume Hood

The layout should follow the workflow so that staff never raise an arm over the Piranha solution.

\section{REFERENCES}

260 [1] Singapore Arms and Explosive Act (Chapter 13), Second Schedule, https://www.police.gov.sg/ /media/spf/files/eservices/arms\%20and\%20explosives\%20act.pdf, (accessed April 2021)

[2] Singapore Arms and Explosives Act (Chapter 13), section 22(2) https://sso.agc.gov.sg/Act/AEA1913\#pr23- (accessed Apr 2021)

[3] Schmidt, H.G. Safe Piranhas: A review. ACS Chemical Health \& Safety (in press)

265 [4] Singapore Police Force, personal communication. 